\title{
Brucellosis-induced avascular necrosis of the hip: a rare case report
}

\author{
Zeinab Mohseni Afshar ${ }^{1}$, Arefeh Babazadeh ${ }^{2}$, Soheil Ebrahimpour ${ }^{2}$, Maria Shirvani ${ }^{1}$, \\ Mohammad Hossein Zamanian ${ }^{1}$, Mohammad Barary², and Ronak Miladi ${ }^{1}$ \\ ${ }^{1}$ Kermanshah University of Medical Sciences \\ ${ }^{2}$ Babol University of Medical Science
}

November 25, 2021

\begin{abstract}
A 28-year-old female with a chief complaint of right lower limb claudication for two weeks was admitted into the infectious disease ward. Because of the unreversible damage to the head of her right femur, a total hip replacement surgery was suggested for this patient.
\end{abstract}

\section{Introduction}

Brucellosis is a widespread zoonotic infection that continues to be a significant public health problem. Brucellosis can affect virtually any system of the body $[1,2]$. The most common complication of brucellosis is osteoarticular system involvement, with a $10-80 \%$ prevalence. However, this infection rarely affects the hip $[1$, 3]. The three most common forms of osteoarticular involvement of this pathogen are spondylitis, sacroiliitis, and peripheral arthritis, including monoarthritis, oligoarthritis ([?] 4 joints), or polyarthritis ([?] 5 joints) [4, 5]. As stated, although the hip joint may be involved in this infection, brucellosis-induced avascular necrosis of the hip has very rarely been reported [6]. Herein, we report a young woman with brucellosis presenting with mono-arthritis of the hip, later diagnosed with avascular necrosis (AVN) destroying the femoral head.

\section{Case report}

A 28-year-old rural woman was admitted into the infectious disease ward with a chief complaint of right lower limb claudication for two weeks. She received anti-brucellosis treatment for one month before her admission. She mentioned no history of trauma, comorbidities, and steroids or alcohol use. On her first admission, her chief complaint was right hip pain, which later was confirmed to be brucellosis. She was discharged from the hospital with a partial improvement of her symptoms after receiving a two-week anti-brucellosis regimen. Then, she was again admitted after her inability to walk without help and a reduced range of movements in her right hip, mainly in flexion and external rotation.

Physical examination on current admission revealed right hip tenderness, a decreased force of the right lower limb, positive Patrick's test, negative Straight Leg Raise (SLR), and reverse SLR tests. Laboratory data were as follows: Wright's titer $=1 / 640 ; 2$-mercaptoethanol $(2 \mathrm{ME})$ titer $=1 / 320$; Coombs Wright titer $=$ 1/1280; erythrocyte sedimentation rate $(\mathrm{ESR})=5 \mathrm{~mm} / \mathrm{h}$; blood culture was negative, anti-cyclic citrullinated peptide (anti-CCP) was positive, antinuclear antibody test (ANA), anti-dsDNA, anti-phospholipid antibody, P-ANCA, C-ANCA, and HLA B27 were negative, and complement C3 and C4 levels were normal.

Pelvic radiography was then performed, showing AVN of the right hip (Figure 1). Right hip ultrasonography (US) was also performed, characterizing a mild effusion and synovial tissue hyperproliferation at the femoral joint capsule. After that, magnetic resonance imaging (MRI) of the hip showed a crescent sign at the proximal portion of the right femoral bone, consistent with AVN of the femoral head (Figure 2). Subsequently, a total hip replacement (hip arthroplasty) surgery was recommended during an initial consultation with an 
orthopedic surgeon. Before and after the surgery, the patient received a three-week standard anti-brucellosis regimen consisting of streptomycin (1g IM daily), doxycycline (100 mg PO BID), and rifampin (600 mg PO daily). Then, she was discharged after her arthralgia had improved and she could walk without aid.

\section{Discussion}

Osteoarticular complications are common in human brucellosis. The sacroiliac (about 80\%) and spinal joints (about 50\%) are the two major affected joints [1]. On the other hand, other osteoarticular complications, such as are bursitis [2], peripheral arthritis, osteomyelitis, and tenosynovitis, are rare [3]. Brucella-induced arthritis can be observed in more than $50 \%$ of patients [3], with the lower limb joints being the most commonly involved ones [7]. Nevertheless, joint destruction has rarely been reported. Thus, lack of early diagnosis and treatment of brucellosis-induced hip arthritis may cause several severe complications, including dislocation and even AVN of the femoral head [6].

Osteonecrosis of the femur head occurs either as an idiopathic process (Legg-Calve-Perthes disease) or as a complication of osteomyelitis, septic arthritis, and toxic synovitis [8, 9]. Usually, AVN can be induced by different etiologies, such as hip dislocation or fracture, long-term use of bisphosphonates and corticosteroid, excessive alcohol use, blood clots, damage to the arteries, Gaucher disease (GD), sickle cell disease (SCD), vasculitis, gout, osteoporosis, pancreatitis, acquired immunodeficiency disease (AIDS), and autoimmune diseases [10]. Many patients have no symptoms in the early stages of AVN. Nonetheless, as the condition progress, pain may become more constant $[10,11]$. Due to the bone and joint collapse, AVN may cause a reduced range of motion in the hip joint. In our case, claudication and disability appeared two months after the patient's first presentation.

Several imaging techniques can be used in detecting AVN. Plain X-ray, computerized tomography (CT) scans, and MRI are accessible options, while MRI has the highest sensitivity and specificity and is considered the gold standard for the diagnosis of AVN [12]. As stated, a permanent bone complication in peripheral arthritis in brucellosis is uncommon, but the delay in diagnosis and treatment of the disease can cause this involvement. Also, this condition may be due to an increased intra-articular pressure related to the infection or the direct involvement of the femoral head with the pathogen. Although the standard three-drug antibrucellosis regimen was initiated for this patient, permanent damage to the right hip joint had occurred, indicating a total hip replacement surgery.

\section{Conclusion}

Since brucellosis may manifest with various symptoms, especially in endemic areas, patients with a suspicion of this disease should be given extra attention. Also, early diagnosis, long-term therapy, and follow-up of these patients are vital in avoiding permanent complications and disability.

\section{Data Availability Statement}

The data that support the findings of this study are available from the corresponding author upon reasonable request.

\section{Acknowledgments}

The authors thank the Department of Infectious Diseases and Tropical Medicine of Babol University of Medical Sciences.

\section{Author contributions}

The case was diagnosed and followed up by RM and ZMA. SE and AB conceived and planned the case report, ZMA, SE, and MHZ wrote the manuscript, and MB edited the first draft and provided substantial revision. The final version was read, corrected, and approved by all authors. All co-authors take full responsibility for the integrity of the case study and literature review.

\section{Conflicts of Interest}


The authors declare that they have no conflict of interest.

\section{Informed consent}

Written informed consent was obtained from the patient for publication of the current case report.

\section{References}

1. Esmaeilnejad-Ganji SM, Esmaeilnejad-Ganji SMR (2019) Osteoarticular manifestations of human brucellosis: A review. World J Orthop 10:54-62. https://doi.org/10.5312/wjo.v10.i2.54

2. Esmaeilnejad-Ganji SM, Roushan MRH, Ebrahimpour S, Babazadeh A (2019) Brucellosis as a rare cause of olecranon bursitis: case-based review. Rheumatol Int 39:2185-2187. https://doi.org/10.1007/s00296-01904414-5

3. Pandit D (2011) Brucella arthritis-an update. Indian J Rheumatol 6:75-79

4. Ebrahimpour S, Bayani M, Moulana Z, Roushan MRH (2017) Skeletal complications of brucellosis: A study of 464 cases in Babol, Iran. Casp J Intern Med 8:44-48

5. Dashti AS, Karimi A (2013) Skeletal involvement of Brucella melitensis in children: A systematic review. Iran J Med Sci 38:286-292

6. Salarvand S, Nazer MR, Shokri S, et al (2012) Brucellosis-induced avascular necrosis of the hip in a middle-aged person. Iran J Public Health 41:86-88

7. Liu M, Zhang W, Li X, et al (2015) Brucella arthritis of the hip: Case report. Radiol Infect Dis 2:51-53. https://doi.org/10.1016/j.jrid.2015.03.001

8. Chaudhry S, Phillips D, Feldman D (2014) Legg-calvé-perthes disease an overview with recent literature. Bull Hosp Joint Dis 72:18-27

9. Vidigal Jr. EC, Vidigal EC, Fernandes JL (1998) Avascular necrosis as a complication of septic arthritis of the hip in children. Int Orthop 21:389-392. https://doi.org/10.1007/s002640050192

10. Tripathy S, Goyal T, Sen R (2015) Management of femoral head osteonecrosis: Current concepts. Indian J Orthop 49:28. https://doi.org/10.4103/0019-5413.143911

11. Lespasio MJ, Sodhi N, Mont MA (2019) Osteonecrosis of the Hip: A Primer. Perm J 23:. https://doi.org/10.7812/TPP/18-100

12. Stoica Z, Dumitrescu D, Popescu M, et al (2009) Imaging of avascular necrosis of femoral head: familiar methods and newer trends. Curr Heal Sci J 35:23-8. https://doi.org/10.1007/s002640050192

Figure legends

Figure 1. Pelvic radiography indicating AVN of the right hip

Figure 2. MRI of the hip depicting a crescent sign at the proximal portion of the right femoral bone, suggesting AVN of the right hip joint 

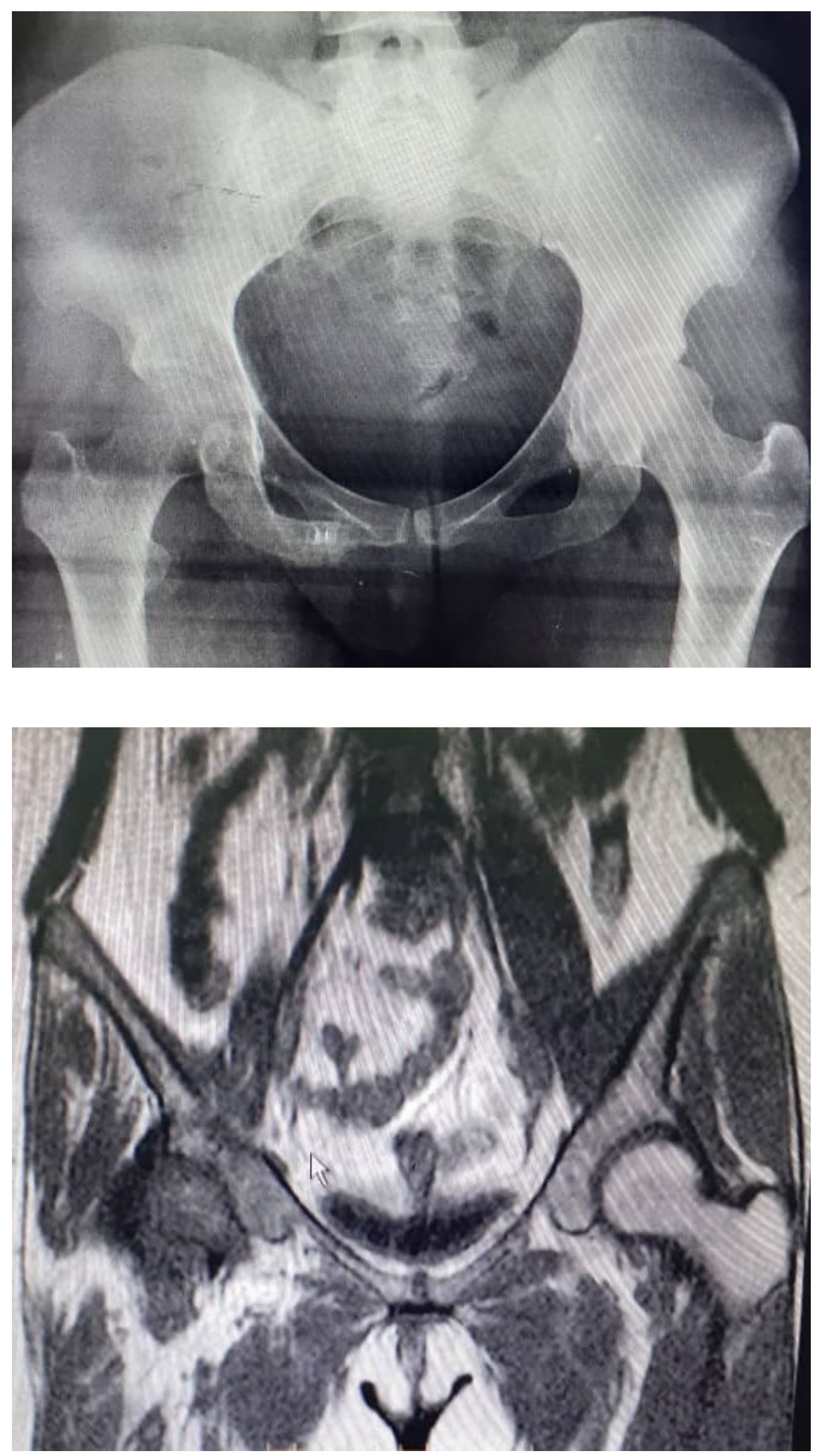